\title{
Connaissances de la Bilharziose Urinaire en Milieu Rural Ivoirien: Etude de Cas à Bamoro et N'guessan-Pokoukro (District Sanitaire de Bouaké)
}

\author{
Ahossi Nicolas Brou, \\ Institut des Sciences Anthropologiques de Développement, \\ UFR des Sciences de l'Homme et de la Société, Université Félix \\ Houphouët-Boigny, Abidjan, Côte d'Ivoire
}

Doi:10.19044/esj.2019.v15n30p113 URL:http://dx.doi.org/10.19044/esj.2019.v15n30p113

\section{Résumé}

La schistosomiase ou bilharziose est l'une des cinq maladies tropicales négligées les plus courantes, observées en Afrique, notamment en Côte d'Ivoire. La présence d'eau douce semble être l'un des facteurs privilégiés de l'existence et de la pérennité des maladies hydriques, notamment les Bilharzioses, une Maladie Tropicale Négligée (MTN) en Côte d'Ivoire. L'objectif de la recherche est de comprendre les facteurs explicatifs qui favorisent la pérennité des Bilharzioses, notamment la Bilharziose urinaire. De manière spécifique, il s'agit d'appréhender les connaissances qu'ont les populations de Bamoro et Nguessan-Pokoukro de la Bilharziose urinaire. La démarche méthodologique a combiné une approche qualitative et quantitative afin de recueillir des données auprès de trois catégories de personnes (élèves du primaire, jeune et adultes). Au terme de l'étude, il apparait que la connaissance de la Bilharziose urinaire se présente sous différentes formes. Chacune des tranches d'âge ou catégorie sociale se représente la maladie en fonction de leurs connaissances des caractéristiques épidémiologiques de cette dernière. Une modification de ses caractéristiques entraînerait certainement une modification de la perception de la maladie.

Mots clés : Bilharziose urinaire, Connaissances, Eau douce, Maladies Tropicale Négligées, Schistosoma hæmatobium 


\title{
Knowledge of Urinary Schistosomiasis in Rural Ivory Coast: Case Study in Bamoro and N'guessan-Pokoukro (Sanitary District of Bouaké)
}

\author{
Ahossi Nicolas Brou, \\ Institut des Sciences Anthropologiques de Développement, \\ UFR des Sciences de l'Homme et de la Société, Université Félix \\ Houphouët-Boigny, Abidjan, Côte d'Ivoire
}

\begin{abstract}
Schistosomiasis or bilharzia is one of the five most common neglected tropical diseases observed in Africa, particularly in Côte d'Ivoire. The presence of fresh water seems to be one of the key factors in the existence and sustainability of waterborne diseases, including Bilharzia, a Neglected Tropical Disease (NTD) in Côte d'Ivoire. The objective of the research is to understand the explanatory factors that promote the survival of Bilharzia, especially urinary bilharziasis. Specifically, it is a question of apprehending the knowledge which the populations of Bamoro and Nguessan-Pokoukro of the bilharziosis urinary. The methodological approach combined a qualitative and quantitative approach to collect data from three categories of people (primary, youth and adult students). At the end of the study, it appears that the knowledge of urinary bilharziasis comes in different forms. Each age group or social category represents the disease according to their knowledge of the epidemiological characteristics of the latter. A change in its characteristics would certainly result in a change in the perception of the disease.
\end{abstract}

Keywords: Urinary bilharziasis, Freshwater, Neglected tropical diseases, Knowledge, Schistosoma hæmatobium

\section{Introduction}

Le développement de tout pays se fait dans le but de garantir le bien être des individus dont la santé est une des composantes essentielles. Malheureusement, les maladies tropicales négligées qui sont un groupe de maladies évitables et curables, affectent 1,5 milliard de personnes, dont $40 \%$ vivent en Afrique. (Uniting to Combat NTDs, 2016). Ces maladies touchent les populations les plus pauvres et les plus vulnérables qui vivent dans les zones reculées d'Afrique. Elles défigurent et provoquent des handicaps, empêchent les enfants d'aller à l'école et les parents de travailler - limitant 
leur potentiel et laissant les habitants prisonniers de la pauvreté. (Uniting to Combat NTDs, 2016).

La schistosomiase ou bilharziose est l'une des cinq maladies tropicales négligées les plus courantes, observées en Afrique, notamment en Côte d'Ivoire. La Côte d'Ivoire, comme la plupart des pays en émergence, a élaboré des projets de développement de l'eau; cela s'est effectué dans le cadre de sa politique économique basée sur l'agriculture. Or les maladies transportées par l'eau, apportées ou répandues par ces projets de développement peuvent aussi faire obstacle à l'aboutissement de ces projets. Cet obstacle se manifeste par l'infestation des populations riveraines. Ces dernières, dans leurs activités quotidiennes se mettent en contact avec l'eau soit pour la nage, la pêche, la lessive ou l'agriculture. La présence d'eau douce semble être un facteur privilégié de l'existence et de la pérennité des maladies hydriques, notamment les Bilharzioses, une Maladies Tropicale Négligées (MTN) en Côte d'Ivoire. En zone rurale, et particulièrement dans le district sanitaire de Bouaké, les Bilharzioses sont toutes aussi présentes. Selon plusieurs résultats des études diagnostiques et épidémiologiques, effectuées depuis 1995, la prévalence de la Bilharziose urinaire s'élève à $43,8 \%$ et celle de la forme intestinale à 1,2 $\%$. En effet, les résultats épidémiologiques ont montré que les schistosomes sont en pleine expansion en Côte d'Ivoire. (N.K, ELIEZER, 1997)

Aujourd'hui, malgré un taux de couverture acceptable en matière de traitement $^{1}$, la maladie demeure une affection à prendre au sérieux. . Les zones rurales semblent être les plus exposées par la présence d'eau douce. Aussi, au regard de cet état de fait, une attention particulière nécessite d'être accordée aux villages de Bamoro et de N'guessan-Pokoukro, deux (2) zones connues pour l'endémicité de la maladie. Il est impératif de rechercher les attitudes et comportements des populations qui concourent au maintien ou à l'intensification de la maladie.

La présence étude a pour objectif de comprendre les facteurs explicatifs qui favorisent la pérennité des Bilharzioses, notamment la Bilharziose urinaire. De manière spécifique, il s'agit d'appréhender les connaissances qu'ont les populations de Bamoro et Nguessan-Pokoukro de la Bilharziose urinaire.

\section{1- Methodologie}

\section{1- Localisation de la zone d'étude}

L'étude a pour cadre le District Sanitaire de Bouaké, à l'intérieur duquel deux villages ont été choisis. Ce sont les villages de Bamoro et de N'Guessan-Pokoukro.

\footnotetext{
${ }^{1}$ Le taux de couverture a augmenté, passant de $18 \%$ en 2015 à $84 \%$ en 2016.
} 
Le District Sanitaire de Bouaké ou du District Saniataire du Gbeke se situe dans le département de Bouaké au centre de la Côte d'Ivoire. Sa population est constituée en majorité d'autochtones Baoulé. L'on y retrouve également divers groupe ethniques aussi bien de la Côte d'Ivoire que de pays étrangers d'Afrique et du reste du monde.

\section{2- Techniques et outils de collecte des données}

L'étude a nécessité la combinaison d'une méthodologie de type mixte. Essentiellement qualitative, l'étude a également intégré un aspect quantitatif afin de relever des points de convergences et de divergence selon les catégories sociales retenues (enfants ou élèves du primaire, jeunes et adultes).

La recherche documentaire a permis la lecture d'ouvrages et revues scientifiques en médecine (santé publique), en sciences sociales, des rapports sur la santé en général et sur les Bilharzioses en particulier.

Des entretiens semi-directif ont eu lieu avec les personnels médicaux et paramédicaux afin d'obtenir des données sur la présence de la maladie dans les villages d'étude, les facteurs explicatifs selon le corps médical, les symptômes, les techniques de dépistage, la prévention et le traitement;

Quant à l'observation directe, elle nous a permis d'apprécier des pratiques sociales sur les sites d'étude Ce sont, entre autre la consommation d'eau pour les besoins domestiques, les pratiques de la baignade, l'existence de toilettes dans les cours ou autours des concessions etc. Toutes ces réalités ont trait à l'environnement socio-sanitaire ainsi qu'aux comportements sanitaires des populations enquêtées.

Pour l'enquête individuelle, nous avons élaboré un questionnaire constitué en majorité de questions fermées avec des propositions de réponses. A cet effet, un échantillon, basé sur la méthode probabiliste, a été défini afin de réaliser l'étude au sein des populations des villages d'enquête en fonction du niveau d'étude et de la catégorie sociale (enfants, jeunes et adultes). Chacun des enquêtés à partir de cette méthode aléatoire avait ainsi la possibilité de figurer dans l'échantillon. Ainsi, l'échantillon était composé des élèves des écoles primaires, les jeunes et les adultes de chacun des villages ; toutes ces personnes appartiennent soit au sexe masculin, soit au sexe féminin. Les élèves du primaire ont l'âge qui varie entre 9 et 15 ans. Les jeunes sont pris en compte dans la tranche d'âge de 15 à 30 ans, quant aux adultes, ils ont l'âge qui varie de 30 à plus de 70 ans.

Il est important de préciser que le choix de ces trois tranches d'âge s'est opéré à partir de constats. En effet, les élèves du primaire, selon les infirmiers des villages rencontrés, semblent être les plus exposés à la maladie. La raison principale évoquée est celle de l'intérêt particulier que ces élèves ont pour les baignades dans les cours d'eau des villages. Quant aux jeunes, certains d'entre eux effectuent des activités agricoles notamment les cultures 
de riz dans les bas-fonds situés aux alentours des villages. Or le contact permanent avec une eau infectée peut provoquer la maladie. Le choix s'est également porté sur les adultes à cause de la durée de vie du parasite dans un corps déjà contaminé et dont les soins seraient négligés. Précisons enfin, qu'étant donné que les groupes scolaires sont fréquentés par les enfants issus des villages d'étude aussi bien que ceux provenant des villages environnants, nous n'avons accordé la priorité aux élèves issus des villages sous-études. La taille de l'échantillon a été répartie entre les trois tranches d'âge retenues (élèves du primaire, jeunes et adultes).

Tableau 1 : Taille de l'échantillon

\begin{tabular}{|l|c|c|}
\hline \multicolumn{1}{|c|}{ Village } & Bamoro & N'guessan-Pokouko \\
\hline Position & & 660 \\
\hline Population Générale & 1570 & 7 \\
\hline Population obtenue & 7 & 46 \\
\hline Total & 110 & 156 \\
\hline
\end{tabular}

Source : Tableau produit par nous-même

Tableau 2: Taille de l'échantillon à Bamoro.

\begin{tabular}{|c|c|c|c|c|c|c|}
\hline \multirow[b]{2}{*}{ Tranche d'âge } & \multicolumn{3}{|c|}{ Masculin } & \multicolumn{3}{|c|}{ Féminin } \\
\hline & $\begin{array}{c}\text { Elève } \\
\text { du } \\
\text { Primaire }\end{array}$ & Jeunes & Adultes & $\begin{array}{c}\text { Elève } \\
\text { du } \\
\text { Primaire }\end{array}$ & Jeunes & Adultes \\
\hline$\%$ Choisi & 45 & 25 & 30 & 45 & 25 & 30 \\
\hline Population obtenue & 28 & 15 & 06 & 20 & 15 & 17 \\
\hline Total & \multicolumn{3}{|c|}{52} & \multicolumn{3}{|c|}{58} \\
\hline TOTAUX & \multicolumn{6}{|c|}{110} \\
\hline
\end{tabular}

Source : Tableau produit par nous-même

Tableau 3: Taille de l'échantillon à N'GUESSAN-POKOUKRO

\begin{tabular}{|c|c|c|c|c|c|c|}
\hline & \multicolumn{3}{|c|}{ Masculin } & \multicolumn{3}{|c|}{ Féminin } \\
\hline d'âge & $\begin{array}{c}\text { Elève } \\
\text { du } \\
\text { Primaire }\end{array}$ & Jeunes & Adultes & $\begin{array}{c}\text { Elève } \\
\text { du } \\
\text { Primaire }\end{array}$ & Jeunes & $\begin{array}{l}\text { Adulte } \\
\mathrm{S}\end{array}$ \\
\hline$\%$ Choisi & 45 & 25 & 30 & 45 & 25 & 30 \\
\hline Population obtenue & 10 & 15 & 06 & 11 & 06 & 8 \\
\hline Total & \multicolumn{3}{|c|}{21} & \multicolumn{3}{|c|}{25} \\
\hline TOTAUX & \multicolumn{6}{|c|}{46} \\
\hline
\end{tabular}

Source : Tableau produit par nous-même

\section{2- Collecte et analyse des données}

L'étude a été réalisée grâce à une combinaison d'approches qui se résument en des visites exploratoires, des entretiens avec les autorités coutumières des villages, les différents responsables des écoles et le personnel de santé (infirmiers, agent de santé communautaire). L'étape suivante a été celle de l'administration du questionnaire. Elle s'est effectuée aussi bien 
pendant la journée que la soirée. En effet, il a fallu profiter des soirées pour soumettre quelques questionnaires à ceux qui, pendant la journée étaient au champ.

Pour les élèves du primaire, les Directeurs des écoles primaire de Bamoro nous ont autorisé à les rencontrer dans leurs différentes classes et ce, pendant les heures creuses (récréations, ...). Quant à N'Guessan-Pokoukro, la direction de l'école primaire a autorisé les élèves choisis par nous, à se regrouper à l'intérieur de la cantine scolaire. Nous avons ainsi pu administrer le questionnaire. Pour les jeunes et les adultes, nous avons pu les rencontrer à différents espaces d'occupation notamment, à l'infirmerie pendant les jours d'affluence et même sur des lieux de contamination (petit barrage, étang...) Enfin, précisons qu'il nous a fallu quelques fois l'aide d'un guide/interprète afin de mieux administrer le questionnaire dans la langue locale des enquêtés (le Baoulé en général). Ajoutons que toutes les personnes enquêtées ont au moins un mois de présence permanente dans le village.

L'analyse des données recueillies au cours de l'enquête de terrain a utilisé deux méthodes. Il s'agit de la méthode descriptive et de la méthode comparative. L'analyse descriptive a pour but de montrer les différentes proportions relatives aux observations en fonction des différentes catégories sociales retenues en vue de leurs interprétations. Le choix de la méthode comparative répond à une logique. C'est de rechercher et d'analyser les différences ou les ressemblances existant entre les situations observées dans chacun des deux villages d'étude.

De fait, il nous est apparu nécessaire de saisir la connaissance des populations des sites de l'étude relativement à la Bilharziose urinaire. Cette connaissance semble être différenciée selon le contexte socioculturel. La connaissance, dans le cadre de la présente étude se traduirait par l'appréhension des caractérisations locales qui se rapporte à la maladie (nosologie, symptôme, étiologie, mode de transmission et les conséquences) selon les communautés d'étude.

\section{2- Resulats}

Précisions que dans le cadre de cette présentation, il sera uniquement fait mention des données quantifiées. Pour rappel, le recueil des données s'est effectué dans deux zones rurales. Aussi, apparaît-il opportun de montrer les connaissances des populations de ces zones d'études au sujet de la Bilharziose urinaire en fonction des catégories sociales retenues: enfants (élèves du primaire), les jeunes et des adultes.

Evoquer dans le cadre de cette étude, l'idée de la connaissance de la maladie, c'est se référer aux différentes caractéristiques que les populations d'enquête relient ou attribuent à la Bilharziose urinaire. Cette connaissance est 
de fait liée aux caractéristiques épidémiologiques de la maladie ainsi qu'aux considérations socioculturelles liées à cette dernière.

\section{1- Le contexte ethno-nosologique}

En Afrique en général et en Côte d'Ivoire en particulier, la maladie est sujette à des interprétations sociales. La dénomination des maladies n'échappe pas à cette règle. Cette dénomination est en fait un ensemble d'éléments socioculturels ou même naturels qui ont des liens avec la maladie.

La présente étude s'est réalisée dans deux villages habités par la communauté Baoulé, une ethnie localisée principalement au centre de la Côte d'ivoire. Toutefois, des allogènes y vivent. Ce sont différentes ethnies de la Côte d'Ivoire (Bété, Sénoufo, Attié...) ainsi que d'autres pays étrangers d'Afrique (Mali, Burkina-Faso...).

Lors des enquêtes, en terme de connaissance nominale de la maladie, l'appellation qui est revenue à maintes reprise, est celle de «MIEH YAH » (mie 'ya) à Bamoro ou « MIE WAH » (mie'wa) à N'guessan Pokoukro.

La légère variation dans la prononciation du nom serait due aux différents groupes de baoulé rencontrés dans les deux villages d'étude. En effet, à Bamoro, nous avons des Baoulé du groupe Farhi-Swafouê tandis qu'à Nguessan-Pokoukro ce sont des Baoulé du groupe Walebo.

Toutefois, la traduction littérale de « mieh yah » ou « mie wah « donne « la douleur de l'urine ou l'urine ou l'urine douloureuse ». L'évocation de l'urine douloureuse émane de la légère douleur à la miction ainsi qu'à la parution d'une coloration rouge-brique.

Cependant s'en tenir à cette traduction littérale, c'est courir le risque de confondre la maladie avec d'autres infections liées aux urines ou au sexe. Les appellations dites moderne et traditionnelle de la maladie existent dans l'ensemble des villages sous-étude. Pour l'appellation moderne, il s'agit de la Bilharziose urinaire. Quant à l'appellation traditionnelle, elle varie au niveau de la prononciation.

Ainsi, à Bamoro, l'on prononce «MIEYAH » et à N'guessanPokoukro l'on prononce plutôt «MIEWAH». Cependant, si ces deux appellations existent, elles ne sont pas connues de tous les enquêtés. Le tableau ci-après illustre nos propos.

Tableau 4: Identification moderne et traditionnelle de la maladie

\begin{tabular}{|l|c|c|c|c|}
\cline { 2 - 5 } \multicolumn{1}{c|}{} & \multicolumn{2}{c|}{ BAMORO } & \multicolumn{2}{c|}{ N'GUESSAN-POKOUKRO } \\
\hline Valeur Dénomination & Effectif & $\%$ & Effectif & $\%$ \\
\hline Bilharziose & 26 & 43,33 & 20 & 62,50 \\
\hline Miewah ou Mieyah & 10 & 16,67 & 07 & 21,88 \\
\hline Bilharziose et Miewah ou Mieyah & 03 & 05 & 03 & 09,37 \\
\hline sans réponse & 21 & 35 & 02 & 06,25 \\
\hline total & 60 & 100 & 32 & 100 \\
\hline
\end{tabular}

Source : Tableau produit par nous-même 
Il ressort que le nombre de personnes connaissant soit l'appellation moderne ou traditionnelle de la maladie ou soit les deux (appellation) varie d'un village à l'autre.

A Bamoro, sur l'ensemble de la population enquêtée, il apparaît que l'appellation traditionnelle de la maladie «Mieyah » est peu connue de cette population. Quant à l'appellation moderne «Bilharziose » elle est connue par près de la moitié de cette même population. Cela se remarque à partir de sonnées chiffrées du tableau ci-dessus. En effet, pour 60 personne enquêtées à Bamoro, seulement 10 ne connaissent que la dénomination de la maladie dans leur ethnie soit $16,67 \%$ de l'échantillon total. Par contre 26 personnes interrogées ont pu dénommer la maladie en français. Ce qui représente 43,33 $\%$ de l'échantillon. Notons une proportion élevée de sans-réponses, 21 personnes soit $35 \%$ de l'échantillon total. Celle-ci provient de la population des élèves du primaire. En effet, au moment de l'enquête, l'enseignement sur les Bilharzioses n'était pas encore abordé. Ce qui a priori justifierait leur méconnaissance de la maladie.

A N'guessan-Pokoukro, sur l'ensemble de la population enquêtée, il apparaît également que l'appellation traditionnelle de la maladie "Miewah » est peu connue. Seule l'appellation moderne «Bilarziose » prédomine. Les données chiffrées du tableau ci-dessus nous éclairent à sujet. En effet, pour 32 personnes enquêtées à N'guessan-pokoukro seulement 7 personnes ne connaissent que la dénomination de la maladie dans leur ethnie soit 21, $87 \%$ de l'échantillon total. Par contre 20 personnes ne connaissent que l'appellation moderne. Ce qui représente $62,50 \%$ de l'échantillon. Enfin, seulement deux (2) enquêtés soit $6,25 \%$ de l'échantillon n'ont donné de réponse.

\section{2- Connaissance de la Bilharziose urinaire.}

Il s'agit de déterminer les connaissances de la Biharziose urinaire au sein des populations de Bamoro et de N'guessan-Pokoukro.

Cette détermination s'effectue à partir de la connaissance des caractéristiques épidémiologiques de la maladie selon les communautés des sites de l'étude.

Celles qui ont été retenues pour cette étude sont entre autre, les symptômes de la maladie, les facteurs étiologiques liées à la maladie, les modes de contamination et l'impact ou les conséquences qui en découlent.

Ici, il est question de montrer la connaissance de la maladie en fonction des différentes catégories sociales retenues au niveau de chacun des villages sous étude. Ce sont des élèves du primaire, des jeunes et des adultes à Bamoro tout comme à Nguessan-Pokoukro. 


\subsection{1- Le cas de Bamoro}

Selon les différentes statistiques obtenues, les symptômes de la Bilarziose urinaire sont connus de la plupart de la population de Bamoro. L'hématurie ou la présence anormale du sang dans l'urine est citée comme le signe le plus évident de la maladie. En effet, sur 36 personnes de l'échantillon ayant une connaissance de ce symptôme, 17 soit $28,32 \%$ sont des élèves du primaire ; 11 sont des jeunes soit 18,34\% et seulement 8 sont des adultes soit $13,34 \%$ de l'échantillon.

Le reste de cette population qui représente $40 \%$ de l'échantillon total (24 personnes) affirme n'avoir jamais contracté la maladie. Ce qui justifierait la méconnaissance du signe clinique. Dans ce groupe, nous avons 11 élèves du primaire, 9 jeunes et seulement 4 adultes. Ces chiffres attestent le fait que l'hématurie est assez connue de l'ensemble de la population comme un symptôme de la maladie. Qu'est-il des facteurs étiologies ou des causes de la maladie selon les enquêtés?

Les données statistiques obtenues indiquent également que la cause de la maladie est connue de la majorité de la population enquêtée. En effet, le parasite de la Bilharziose, même s'il n'est pas connu de nom, ce parasite est présenté comme un microbe vivant dans l'eau de surface qui est à l'origine de la maladie.

Ainsi, sur 60 personnes enquêtées à Bamoro, 43 ont-elles une connaissance de cette cause. Ce qui représente $71 \%$ de l'échantillon réparti de la manière suivante 17 élèves du primaire (28,34\%de l'échantillon). QU'en est-il du mode de contamination?

La majorité de la population enquêtée à Bamoro connaît les modes de contamination de la maladie. Le contact avec une eau infectée est la principale voie de contamination.

Ainsi, sur les 60 personnes enquêtées, 33 ont pu citer ce mode de contamination soit $55 \%$ et 5 adultes $8,33 \%$. Seulement 27 personnes ont affirmé ne pas connaître ce mode de contamination ( $45 \%$ de l'échantillon).

Cependant, il faut ajouter aux différentes réponses données d'autres voies de contamination à envisager selon les enquêtés. En effet, pour certains adultes, en plus du contact avec une eau infectée, l'on pourrait contracter la maladie en ayant des relations sexuelles avec une personne atteinte de la bilharziose urinaire.

Quant aux conséquences liées à la maladie, elles sont quasiment ignorées par l'ensemble de la population. Au nombre des conséquences, nous pouvons citer la stérilité à long terme, la destruction lente des reins, la prostate etc. Cependant, 16 personnes parmi les enquêtées ont affirmé connaître les conséquences de la maladie. Elles ont cité le décès du malade non traité comme la conséquence la plus évidente, la stérilité et des cas probables de problèmes de reins. 
Ce groupe de personnes représente 26,66 \% de l'échantillon. Nous avons 7 élèves du primaire, 6 jeunes et 3 adultes. Ainsi, plus de la moitié de l'échantillon qui représente $73,34 \%$ a affirmé ne pas connaître les conséquences liées à la maladie. Ce sont 21 élèves du primaire, 14 jeunes et 9 adultes.

\subsection{2- Le cas de N'guessan-Pokoukro.}

Tout comme à Bamoro, c'est un ensemble de caractéristiques épidémiologiques qui nous servira de support dans l'approche de la connaissance de la maladie.

Les différentes statistiques nous indiquent que les symptômes de la maladie sont connus de l'ensemble des enquêtés ; d'ailleurs, ceux-ci citent l'hématurie comme le signe clinique le plus évident de la maladie. Comme autre signe, il faut noter de légères démangeaisons après bain dans une eau de surface.

Ainsi, sur les 32 personnes de l'échantillon ayant participé à l'enquête, 28 ont affirmé avoir une connaissance des symptômes ; ce qui représente 87,5 $\%$ du total. Ce sont 14 élèves du primaire soit 43,76 \%, 7 jeunes $(21,87 \%)$ et 7 adultes $(21,87 \%)$.

Seulement 4 personnes, toutes des jeunes, ont répondu ne pas connaître les symptômes de la maladie. La raison évoquée est à lier au fait qu'elles affirment n'avoir jamais contracté la maladie.

Pour les causes de la maladie, ici également, nous pouvons affirmer que l'ensemble de la population enquêtée a au moins une connaissance de la cause de la maladie. C'est un microbe aquatique que l'on retrouve dans l'étang du village.

Une fois de plus, seules 4 personnes n'ont pas donné de réponse sur l'ensemble des enquêtes. En effet, concernant la cause de la maladie, 28 personnes représentant $87,5 \%$ de l'échantillon total ont affirmé la connaitre. Ce sont toujours 14 élèves du primaire, 7 jeunes et 7 adultes.

Quant aux modes de contamination, l'on constate qu'il s'agit du contact avec un cours d'eau infecté. Ce sont toujours les mêmes personnes déjà mentionnées plus haut qui ont affirmé connaître ce mode de contamination de la maladie.

Cependant, il y a lieu de préciser que d'autres voies de contaminations ont été citées au cours de l'enquête. En effet pour certains jeunes, les relations sexuelles avec une personne déjà atteinte par la maladie sont des risques d'infection. Aussi, la maladie serait-elle transmissible par voie sexuelle.

Il faut remarquer que contrairement aux autres caractéristiques épidémiologiques de la maladie, les conséquences semblent être peu connues. En effet, plus de la moitié de la population enquêtée a affirmé ne pas connaître les conséquences liées à la maladie. 
L'on note que seulement 13 personnes sur les 32 que compte l'échantillon connaissent les conséquences de la maladie. Elles citent par exemple le décès à long terme du malade comme la conséquence inévitable en l'absence d'un traitement curatif.

D’autres, en occurrence, des jeunes et des adultes énoncent ces décès comme une conséquence probable de la maladie. Quant aux jeunes du primaire même s'ils ont affirmé en connaître, ils n'ont pu les citer.

Ce total de 13 personnes représente 40,63\% de l'échantillon contre 19 qui ont affirmé ne pas connaître les conséquences liées à la maladie soit 59,37 \% .

En somme, il nous apparait que la maladie est différemment connue au sein de la communauté de N'guessan-Pokoukro.

\section{Discussion}

\section{1- Brève synthèse}

L'étude montre que les caractéristiques épidémiologiques de la Bilharziose urinaire sont connues de façon différente par chacune des catégories sociales retenues.

Chez les élèves du primaire de Bamoro à partir d'un ensemble de données recueillies au cours de l'enquête, l'on peut évoquer cette connaissance à partir de certaines caractéristiques.

Pour eux, l'hématurie est le signe clinique le plus évident de la maladie. La Bilharziose urinaire est une maladie dont l'agent pathogène est un microbe aquatique ; ses modes de contamination sont connus de la majorité des élèves du primaire. Ils pensent que les contacts avec une eau infectée sont les voies principales de contamination. Cependant, contrairement aux autres caractéristiques épidémiologiques de la maladie, les conséquences semblent être ignorées par les élèves du primaire de Bamoro.

A N'guessan-pokoukro, pour les élèves du primaire, la maladie est perçue à partir d'un ensemble de connaissances des caractéristiques épidémiologiques de cette dernière.

Tous les élèves du primaire interrogés ont cité l'hématurie comme le signe clinique de la maladie. Pour eux, la Bilharziose urinaire est une maladie dont la cause principale est un microbe vivant dans une eau douce. La maladie est contractée au cours d'une activité ayant trait à l'eau. C'est par exemple la baignade, la pêche, la lessive. Toutefois, en qui qui concernent les conséquences de la maladie, aucun élève du primaire n'a pu en citer.

$\mathrm{Au}$ niveau des jeunes de Bamoro, la maladie est appréhendée à partir d'un ensemble de données. La majorité des jeunes interrogés pense que l'hématurie est le signe clinique le plus évident de la maladie. La cause principale de la Bilharziose urinaire est un microbe vivant dans l'eau douce ; le contact avec une eau infectée est un risque de contraction de la maladie. Cependant, certains pensent que la voie sexuelle serait aussi un mode de contamination. Quant aux 
conséquences liées à la maladie, il faut noter l'ignorance des jeunes à ce sujet. Cependant, pour certains, le décès du malade est une conséquence inéluctable pour ce dernier s'il n'est pas soigné tôt.

Chez la population jeune de N'guessan-Pokoukro, la maladie est également connue en fonction des caractéristiques émanent de celle-ci. Le symptôme le plus indicatif de la maladie est l'hématurie ou la couleur du sang. La Bilharziose urinaire est une maladie dont la cause est liée à la présence de parasites dans les eaux douces. Certains jeunes pensent que la maladie aurait pour facteur les urines dans ces eaux douces. Ce qui causerait la contraction de la maladie. En effet, l'on contracte la maladie en étant en contact avec une eau de surface ; les activités liées à l'eau seraient de ce fait des facteurs de contaminations.

Quant aux conséquences liées à la maladie, elles semblent être ignorées par la majorité des jeunes. Cependant certains parmi eux n'ont pas hésité à avancer la stérilité comme conséquence inévitable.

Chez les adultes, une fois de plus c'est un ensemble de données qui nous permet d'énoncer leur connaissance de la bilharziose urinaire. L'hématurie est le signe clinique de la maladie. La bilharziose urinaire est une maladie provoquée par un corps étranger dans l'organisme humain. Ce corps étranger a pour habitat les eaux douces du village. La maladie se contracte en étant en contact avec un cours d'eau infecté et ce, à travers la pratique d'un certain nombre d'activités liées à l'eau. Concernant les conséquences, très peu de personnes adultes en ont une idée. Elles sont quasiment ignorées de l'ensemble des adultes rencontrés. Néanmoins ils pensent au décès du malade s'il n'est pas soigné au moment opportun. Certains parmi ces adultes pensent que la maladie aurait aussi pour conséquence la stérilité.

Chez les adultes de N'guessan-pokoukro, très peu ont accepté de répondre aux questions au cours de l'enquête. Ceux qui ont participé à l'étude semblent avoir une idée assez précise de la maladie.

Si pour eux, la maladie est reconnaissable à partir de l'hématurie chez une personne infectée, la cause évidente provient d'un corps étranger résidant dans l'eau de surface ; c'est un microbe.

La maladie se contracte au cours d'un certain nombre d'activité en rapport avec une eau infectée. Cependant, la quasi-totalité des adultes rencontrés ignore la conséquence inévitable tout comme le décès du malade en cas de non traitement efficace.

$\mathrm{Au}$ regard des données ci-dessus résumés, Il ressort de fait que la connaissance de la maladie à Bamoro est essentiellement tributaire des caractéristiques épidémiologiques de la Bilharziose urinaire. En effet, il est à noter que ces différentes connaissances ont trait aux caractéristiques épidémiologiques de celle - ci. Toutefois, elles reflètent les caractéristiques connues par la médecine moderne. De ce qui suit, il apparaît que la bilharziose 
urinaire est perçue différemment au sein de la population de Bamoro. Chacun se représente la maladie en fonction de ses connaissances des caractéristiques épidémiologiques de cette dernière. Les élèves du primaire perçoivent différemment la maladie par rapport aux jeunes. Également, les jeunes ne semblent pas percevoir de la même manière que les adultes.

Contrairement à Bamoro, la population de N'guessan-Pokoukro semble connaître l'ensemble des caractéristiques épidémiologiques de la maladie. Chacune des tranches d'âge rencontrées se représente la maladie en fonction de leur connaissance de ces caractéristiques.

Ici également, quelques-uns ont affirmé que la maladie pourrait être le fait d'un mauvais sort jeté à une tierce personne. Nul ne serait à l'abri d'un tel sort tendant à nuire à un individu.

\section{2- Implication de la connaissance des maladies par les populations}

Les différentes caractéristiques qui favorisent la connaissance de la maladie, contribuent également à la perception sociale de la maladie. Il s'agit surtout des caractéristiques relevant de la cause et de contamination de la maladie. Elle participe de la «construction sociale de la maladie comme le soulignent Y.JAFFRE et J.-P OLIVIER DE SARDAN (1999 p7) « jamais, nulle part, les représentations populaires des maladies ne coïncident avec les représentations savantes, biomédicales ».

L'on a pu constater que pour certains jeunes et adultes de Bamoro, la cause de la maladie est également liée à un mauvais sort que l'on aurait jeté à un individu. Aussi, la maladie serait- elle provoquée par une tierce personne. Le «sorcier » du village en est parfaitement capable. Enfreindre également un interdit lié à l'eau, c'est courir le risque de mécontenter les esprits de cette eau et donc, il serait possible de contracter la maladie.

Pour ce qui est des modes de contamination certains adultes rencontrés ont évoqué le fait que le contact avec une eau infectée n'est pas le seul mode de contamination. Pour eux, les relations sexuelles sont aussi une voie de contamination de la maladie, tout comme le fait de mettre le pied dans les urines d'un malade.

L'étude nosographique a montré également la difficulté à identifier la Bilharziose dans la langue locale. D'ailleurs cette identification connaît des variations dans la prononciation. Mais il est à retenir que la Bilharziose urinaire ou Schistosomiase est connue sous le nom «Mieyah ou Miewah ». Elle est ainsi désignée par rapport à l'urine rouge-brique de la maladie. Ce qui fait dire qu'il s'agit effectivement de la Bilharziose urinaire, une des formes des Bilharzioses en général.

Cette connaissance de la maladie ne diffère pas de celle observée par N. N. ABE (2014). Selon l'auteur, la connaissance de la bilharziose par les populations participe également de la représentation sociale de la maladie par 
les communautés culturelles. Cette représente consiste à décrire le nom, les symptômes et également les causes.

C'est ici le lieu de mentionner que la plupart des personnes interrogées n’ont donné que la forme urinaire de la maladie. Elles semblent ignorer les autres formes telles que la Bilharziose intestinale ou encore la Bilharziose rectale.

En effet, la Bilharziose urinaire est la plus évidente et la mieux perceptible. Cela est surtout dû à l'aspect visible de l'hématurie macroscopique.

En somme, la maladie, du moins, la dénomination de la maladie varie d'une personne à une autre, d'un village à un autre.

Pour A. N. SINDZINGRE (1984), du fait également de la connaissance de la maladie, les représentations de la maladie, ne sont pas généralisables à toutes les sociétés africaines. Selon l'auteur, elles font appel à des systèmes de représentations qui diffèrent même si on observe certains traits largement récurrents, en particulier la prégnance des variables exogènes telles que l'univers physique et humain, des ancêtres et des puissances surnaturelles. Ainsi, les représentations, par exemple, de la santé varient d'une société à une autre, et même, à l'intérieur de la même société, d'un groupe à l'autre.

C.G HELMAN (2000), observe de même une variation des représentations sociales en lien avec la santé dans les sociétés non industrialisées. Pour lui, ces représentations traduisent la vision de l'univers, où monde visible et invisible se côtoient, et où l'ordre social est tributaire de l'harmonie entre ces deux mondes.

Ainsi, de la connaissance de la maladie à sa perception sociale, l'on note qu'elle varie aussi bien au niveau de chacune des tranches d'âge que d'un village à un autre. En effet, la maladie est perçue par l'ensemble des populations en fonction de leur connaissance des caractéristiques épidémiologiques de l'infection. Il apparaît de ce fait que la maladie, si elle est bien présente dans la pensée et la vision des populations, elle ne saurait être connue, ni perçue de manière homogène.

\section{Conclusion}

La présente contribution est issue des données d'étude sur la Bilharziose urinaire réalisée dans deux villages du District Sanitaire qui ont servi de cadre d'étude : Bamoro et N'guessan-pokoukro.

Pour rappel, la schistosomiase (encore appelée schistosomose ou bilharziose) est une maladie parasitaire due aux trématodes du genre Schistosoma (S.), donc 5 espèces sont pathogènes pour l'homme. C'est la deuxième grande endémie parasitaire dans le monde. Selon l'OMS, La schistosomiase est une parasitose chronique provoquée par des vers 
(trématodes) du genre Schistosoma. Au moins 206,5 millions de personnes avaient besoin d'un traitement en 2016. On estime à environ 4 millions d'infestations par an et entre 300000 à 500000 décès par an. Le facteur influençant le développement de la schistosomiase est le contact avec les gites des mollusques et larves notamment dans les eaux douces infectées.

L’objectif de la recherche est d'appréhender les connaissances de la Bilharziose urinaire par les populations. Cette connaissance pourrait expliquer la persistance de la maladie au sein des populations des villages sous-étude.

Pour ce faire, la démarche méthodologique a combiné une approche qualitative et quantitative afin de recueillir des données auprès de trois catégories de personnes (élèves du primaire, jeune et adultes).

Au terme de l'analyse des données, il apparait que la maladie est bien connue par les populations mais de manière différenciée. En effet, la Bilharziose urinaire se présente sous différentes formes selon les catégories sociales ou tranches d'âges identifiées. Leurs diverses connaissances des maladies sont liées aux caractéristiques épidémiologiques de cette dernière.

En somme, au regard de ces représentations sociales, la Bilharziose urinaire ne peut qu'être favorisée, occasionnant sa persistance au sein de la population.

\section{References:}

1. ABBE N'doumy Noél, 2014, «Contribution of Socio-Anthropology in Schistosomiasis Control - TAABO/Côte d'Ivoire» Experiment. J Homeop Ayurv Med 3:144. doi: 10.4172/2167-1206.1000144

2. HELMAN Cecil G, 2000 «Culture, santé et maladie », 4e édition. Londres, Arnold, pp328

3. JAFFRÉ Yannick et OLIVIER DE SARDAN Jean-Pierre, 1999, « La construction sociale des maladies : les entités nosologiques populaires en Afrique de l'Ouest ». Paris, Les Presses universitaires de France, 376 pp. Collection Les champs de la santé.

4. N'GORAN Kouakou Eliezer, 1997, Biodiversité, transmission et épidémiologie de Schistosoma haematobium, Bilharz, 1852 et des schistosomes apparentés en Côte d'Ivoire, Thèse de doctorat en Biologie, Université de Perpignan, France.

5. Uniting to Combat NTDs, 2016, La Côte d'Ivoire et les maladies tropicales négligées.

6. SINDZINGRE Alice Nicole, 1984, «La nécessité du sens: l'explication de l'infortune chez les Senufo, in Augé et Herzlich (éd.), «Le sens du mal. Anthropologie, histoire, sociologie de la maladie, Paris, Éditions des Archives contemporaines. 